\title{
Analysis of transgenic sugarcane overexressing a co-chaperone using RT-qPCR
}

\section{Ana Laura G. Leme*, Giovanna V. Guidelli, Marcelo Menossi}

\begin{abstract}
Sugarcane (Saccharum spp.) is an important crop and is affected by many abiotic stresses, such as drought, the single most important cause of losses in agriculture. Co-chaperones have been found to protect plants from water defict. We identified a sugarcane gene encoding a $D^{\prime}$ co-chaperone and have produced transgenic sugarcane plants overexpressing this protein. These plants will be used to assess the role of $D^{\prime}$ co-chaperones in sugarcane under water deficit stress.
\end{abstract}

\section{Key words:}

sugarcane, co-chaperone, $R T$-qPCR

\section{Introduction}

Sugarcane is an important crop and the main raw material to sugar and ethanol production. Among many biotic and abiotic stresses, drought occupies a central position.

D' proteins are co-chaperones with $\mathrm{J}$ domain (Caplan et al., 1993). Among their functions, D' proteins are involved in many stress responses (Yang et al., 2010). Results using transgenic Arabidopsis showed that overexpression of tobacco D' protein increased the transcription of antioxidant genes involved in the detoxification reactive oxygen species (Xia et al., 2014). Additionally, this protein may participate in water stress control by acting in ABA dependent signaling pathways.

The development of new cultivars combining tools of classical breeding and biotechnology emerges as a promising alternative to increase the productivity of sugarcane.

\section{Results and Discussion}

\section{Total RNA extraction and RNA integrity}

To analyse the $D^{\prime}$ overexpression in transgenic plants, total RNA from 20 independe events was extracted following modified guanidine protocol (Chirgwin et al., 1979). As negative control, six events of plants transformed with empty vector were selected. To confirm RNA integrity, we used denaturing agarose gel $2 \%$.

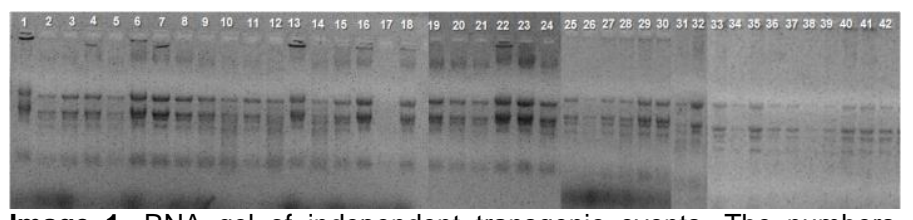

Image 1. RNA gel of independent transgenic events. The numbers indicate the independent events: V1a, V1b, V1c, V2a, V2b, V2c, V4a, V4b, V4c, V6a, V6b, V6c, D'1a, D'1b, D'1c, D'3a, D'3b, D'3c, D'10a, D'10b, D'10c, D'19a, D'19b, D'19c, D'2a, D'5a, D'8c, D'11b, D'16a, D'20c, V3a, V5a, D'4a, D'6a, D'9a, D'12a, D'13a, D'14a, D'15a, D'18a, D'28a, D'60a.

\section{Gene expression analysis using RT-qPCR}

The following data show gene expression levels in transgenic plants and empty vector in relation to a gene encoding ubiquitin as a normalizer.

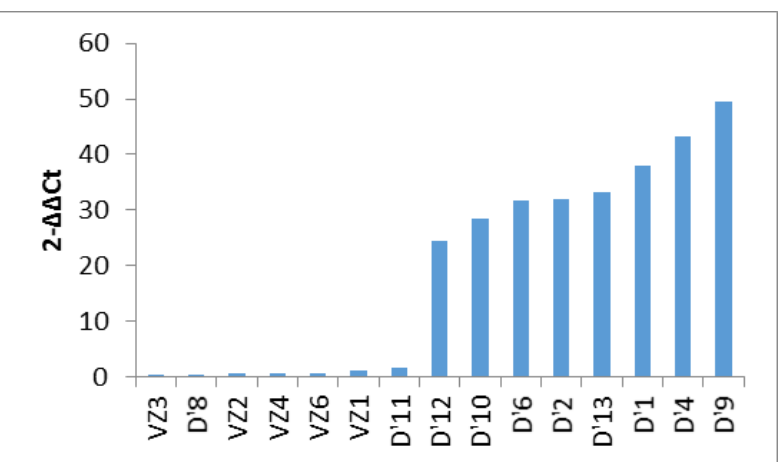

Image 2. Gene expression analysis of D' transgene using $\Delta \Delta C T$ analysis method (Pabinger et al., 2009)

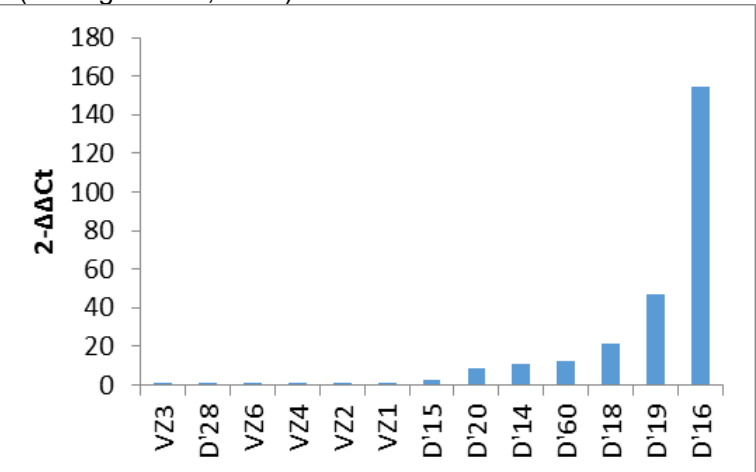

Image 3. Gene expression analysis of D' transgene using $\Delta \Delta C T$ analysis method (Pabinger et al., 2009)

\section{Conclusions}

The transformation of sugarcane plants was effective, producing several independent transgenic events with variable expression levels of D' transgene.

For future analysis, the events D'4, D'9, D'16 e D'19 were seleted to drought stress experiments in greehouse. As negative control we will use empty vector plants.

\section{Acknowledgement}

This work was carried out with the support from CNPq, National Council for Scientific and Technological Development - Brazil.

Caplan et al. Mol. Bio. Cell. 1993 4, 555-563.

Chirgwing et al. Biochemistry. 1979. 18, 5294-5299

Xia et al. Plant Physiology and Biochemistry. 2014. 83, 100-106.

Yang et al. Plant Cell. 2010. 22, 1313-1332 